\title{
Expression of programmed death 1 ligand 1 on periodontal tissue cells as a possible protective feedback mechanism against periodontal tissue destruction
}

\author{
JIEHUA ZHANG ${ }^{*}$, CHIEH-MEI WANG ${ }^{*}$, PING ZHANG ${ }^{*}$, XIAOQIAN WANG, JIAO CHEN, \\ JUN YANG, WANLU LU, WENJIE ZHOU, WENWEN YUAN and YUN FENG \\ State Key Laboratory of Oral Diseases, West China School of Stomatology, \\ Sichuan University, Chengdu, Sichuan 610041, P.R. China
}

Received April 1, 2015; Accepted January 4, 2016

DOI: $10.3892 / \mathrm{mmr} .2016 .4824$

\begin{abstract}
Programmed death 1 ligand 1 (PD-L1) is a negative co-stimulatory molecule in immune responses. Previous reports have indicated that inflammatory cytokines can upregulate the expression of PD-L1 in tumor cells, which in turn suppresses host immune responses. Periodontitis is characterized by persistent inflammation of the periodontium, which is initiated by infection with oral bacteria and results in damage to cells and the matrices of the periodontal connective tissues. In the present study, the expression and function of PD-L1 in periodontal tissue destruction were examined. Periodontal ligament cells (PDLCs) were stimulated by inflammatory cytokines and periodontal pathogens. The expression and function of PD-L1 on the surface of PDLCs was investigated using flow cytometry in vitro. Periodontal disease was induced by the injection of Porphyromonas gingivalis in mouse models. The expression levels of PD-L1 in the periodontal tissues of the mice were analyzed using flow cytometry and immunohistochemistry. PD-L1 was inducibly expressed on the PDLCs by the inflammatory cytokines and periodontal pathogens. The inflammation-induced expression of PD-L1 was shown to cause the apoptosis of activated $\mathrm{T}$ lymphocytes and improve the survival of PDLCs. Furthermore, in the mouse model of
\end{abstract}

Correspondence to: Professor Yun Feng, State Key Laboratory of Oral Diseases, West China School of Stomatology, Sichuan University, 14 Section 3 South Renmin Road, Chengdu, Sichuan 610041, P.R. China

E-mail: fengyunxixi@aliyun.com

*Contributed equally

Abbreviations: PDLCs, periodontal ligament cells; PBMCs, peripheral blood mononuclear cells; P.g, Porphyromonas gingivalis; F.n, Fusobacterium nucleatum; P.i, Prevotella intermedia

Key words: programmed cell death 1 ligand 1, inflammatory cytokine, periodontitis, protective factors experimental periodontitis, the expression of PD-L1 in severe cases of periodontitis was significantly lower, compared with that in mild cases. By contrast, no significant differences were observed between the healthy control and severe periodontitis groups. The results of the present study showed that the expression of PD-L1 may inhibit the destruction of periodontal tissues, indicating the involvement of a possible protective feedback mechanism against periodontal infection.

\section{Introduction}

Chronic periodontitis is characterized by persistent inflammation of the periodontium, which is initiated by infection with oral bacteria and results in damage to cells and the matrices of the periodontal connective tissues (1). According to the latest epidemiological analysis, periodontitis affects up to $50 \%$ of adults in the US (2); it is the leading cause of tooth loss in adults and is shown to positively correlate with life-threatening systemic diseases (3). Although periodontal damage is known to result from the secretion of toxins and generation of reactive oxygen species by periodontal pathogens, the principal clinical feature of periodontitis is the activation of the host immunoinflammatory response $(4,5)$.

The immune response against oral pathogenic bacteria in the periodontium acts as a double-edged sword (6-9). Bacteria can elicit innate and adaptive immune responses, however, the responding inflammatory cytokines and activated inflammatory cells can mediate destruction of the periodontal tissues $(5,10-12)$. The shift in balance between protecting the periodontal tissue and inducing periodontal destruction is caused by the persistent chronic inflammatory response at the periodontal ligament (PDL), a connective tissue located between the cementum and the alveolar bone (13). PDL cells (PDLCs) are an important cell type in the periodontium, and are key in maintaining homeostasis and in the remodeling of periodontal tissues (14). In addition, PDLCs exhibit stem cell properties by inducing chondrogenesis through the growth factors, transforming growth factor (TGF)- $\beta 3$ and bone morphogenetic protein (BMP)-6 (15). PDLCs remodel extracellular matrices through phagocytosis and the release of 
matrix metalloproteinases, and pathological changes in resident PDLCs are positively correlated with periodontitis-associated destructive processes (16).

Programmed cell death 1 ligand 1 (PD-L1) is a transmembrane protein of the B7 family (17). The expression of PD-L1 has been shown to suppress the immune responses elicited by chronic infections in cancer (18). PDL-1 receptors (programmed death-1; PD-1) are constitutively expressed on macrophages, antigen-presenting cells (APCs) and dendritic cells, and are inducibly expressed on activated T cells, B cells, endothelial cells and epithelial cells (19). The binding of PD-L1 with PD-1 can inhibit the activation and proliferation of immune cells, and the secretion of cytokines, which leads to apoptosis of the immune cells. The PDL-1/PD-1 pathway has been demonstrated to be important in the immune evasion and immune tolerance of tumors $(20,21)$. PD-L1 also regulates the development, maintenance and function of induced regulatory T cells (22).

The expression of PD-L1 has been investigated extensively in the majority of types of human cancer, and PD-L1 has been shown to suppress the antitumor immune responses of the host $(21,23)$. Although the expression of PD-L1 in periodontal tissues has been reported by Konermann et al (24), its function in periodontal tissue damage remains to be elucidated. In the present study, PDLCs were used as representatives of periodontal tissue cells to examine the expression of PD-L1 on PDLCs stimulated with inflammatory cytokines and periodontal pathogens in vitro. Furthermore, the association between the expression of PD-L1 and periodontal tissue destruction was investigated in mouse model of experimental periodontitis. The present study aimed to investigate whether PD-L1 was negatively associated with peridontal tissue destruction in vivo. Thus, PD-L1 expression may have potential to be utilized to regulate peridontal tissue destruction.

\section{Materials and methods}

Animals. Twenty male BALB/c mice (6-week-old; 23-25 g) were purchased from the Animal Center of Chinese Academy of Sciences (Shanghai, China) and housed in a specific-pathogen free laminar flow room under constant temperature $\left(25-27^{\circ} \mathrm{C}\right)$, a 12-h light/dark cycle and humidity (40-50\%) with access to food and water ad libitum. All experiments and animal care procedures were approved by the Animal Center of Sichuan University (Sichuan, China). All experimental procedures were approved by the Experimental Animal Committee of the State Key Laboratory of Oral Diseases, West China College of Stomatology, Sichuan University (Chengdu, China).

Isolation and culture of PDLCs. The PDL tissues were obtained from the premolar teeth of three donors, which were extracted for orthodontic purposes at the West China Hospital of Stomatology of Sichuan University. The protocols regarding the use and manipulation of PDL tissues were approved by the Institutional Review Board of West China Hospital of Stomatology, Sichuan University (Chengdu, China) and written informed consent was obtained from each donor. The extracted teeth were rinsed and placed in Dulbecco's modified Eagle's medium (DMEM; GE Healthcare Life Sciences, Logan, UT, USA) supplemented with 1,000 U/ml penicillin and 1,000 $\mu \mathrm{g} / \mathrm{ml}$ streptomycin (Hyclone; GE Healthcare Life Sciences, Logan, UT, USA). The remaining procedures were performed, as described by Arnold et al (25). PDLs attached to the middle third of the root were removed with a curette to avoid contamination with the gingival and apical tissues. The PDL tissues were cut into $\sim 1 \mathrm{~mm}^{2}$ pieces and placed in $25 \mathrm{~mm}^{2}$ culture flasks for cell culture in DMEM medium supplemented with $10 \%$ fetal bovine serum (FBS), $100 \mathrm{U} / \mathrm{ml}$ of penicillin and $100 \mu \mathrm{g} / \mathrm{ml}$ of streptomycin at $37^{\circ} \mathrm{C}$, under $5 \% \mathrm{CO}_{2}$ and $95 \%$ humidity. After reaching confluence of $\sim 75 \%$ (in $\sim 7$ days), the cells were treated with $0.25 \%$ trypsin- $0.1 \%$ EDTA (Hyclone; GE Healthcare Life Sciences) for cell passage. PDLCs at the 5 th or 6th passage were used for the present studies.

Preparation of pathogens and peripheral blood mononuclear cells (PBMCs). The periodontal pathogens Porphyromonas gingivalis (P.g; ATTC33277), Prevotella intermedia (P.i; ATTC25611) and Fusobacterium nucleatum (F.n; ATTC25586) were obtained from State Key Laboratory of Oral Diseases (Chengdu, China) and cultured in brain heart infusion broth (Oxoid Ltd, Basingstoke, UK) under anaerobic conditions at $37^{\circ} \mathrm{C}$ for $48 \mathrm{~h}$. The supernatants of the P.g culture were collected by centrifugation for $20 \mathrm{~min}$ at 9,600 $\mathrm{x} \mathrm{g}$, and stored at $-80^{\circ} \mathrm{C}$. Blood samples $(50 \mathrm{ml})$ were taken from six healthy donors and the PBMCs were isolated using human lymphocyte separation tubes (DAKEWE, China), according to the manufacturer's protocols. The supernatants of the PBMCs were collected by centrifugation at $1,000 \mathrm{x} \mathrm{g}$ for $15 \mathrm{~min}$ and stored at $-80^{\circ} \mathrm{C}$. The full details of the procedure were as described by Lu et al (23). The protocols regarding the use and manipulation of PBMCs were approved by the Institutional Review Board of West China Hospital of Stomatology, Sichuan University (approval no. WCHSIRB-D-2013-039) and written informed consent was obtained from the donors.

Stimulation of PDLCs with inflammatory cytokines. The PDLCs were seeded in a 24 -well plate at a density of $8 \times 10^{4}$ cells/well and were incubated overnight at $37^{\circ} \mathrm{C}$, under $5 \% \mathrm{CO}_{2}$ and $95 \%$ humidity. The cells were then stimulated with $10 \mathrm{ng} / \mathrm{ml}$ interleukin (IL)-1, IL-6, TNF- $\alpha$ or interferon (IFN)- $\gamma, 50 \mathrm{ng} / \mathrm{ml}$ lipopolysaccharide (LPS), a combination of IL-1, IL-6, TNF- $\alpha$ and IFN- $\gamma$ mixed at the ratio of 1:1:1:1 to a final concentration of $10 \mathrm{ng} / \mathrm{ml}$ of cytokines, or periodontal pathogenic bacteria at a PDLC:bacteria ratio of 1:50. The surface expression of PD-L1 on the stimulated PDLCs was measured using flow cytometric analysis. Recombinant human IFN- $\gamma$, IL-1, IL-2, IL-6 and TNF- $\alpha$ were purchased from R\&D Systems (Minneapolis, MN, USA).

Flow cytometry. The pre-treated PDLCs were harvested, washed twice with FCM buffer, comprising phosphate-buffered saline (PBS; ZSGB-BIO, Beijing, China) with 5\% FBS (Hyclone; GE Healthcare Life Sciences) and $0.1 \% \mathrm{NaN}_{3}$ (Sigma-Aldrich), and incubated with either phycoerythrin (PE) mouse anti-human PD-L1 monoclonal antibody (3 $\mu 1$ per sample; dilution, 1:40; BioLegend, Inc., San Diego, CA, USA; cat. no. 329706) or isotype control antibody (3 $\mu 1$ per sample; dilution, 1:40; BioLegend, Inc.; cat. no. 400320) for $30 \mathrm{~min}$ at $4^{\circ} \mathrm{C}$. The stained cells were washed twice with FCM buffer and analyzed using flow cytometry (Beckman Coulter FC500; 
Beckman Coulter, Miami, FL, USA) with Submit 5.2 software (Beckman Coulter).

$T$ cell apoptosis assay. The PDLCs were pre-treated with $10 \mathrm{ng} / \mathrm{ml}$ of TNF- $\alpha$ or IFN- $\gamma$ for $48 \mathrm{~h}$ to induce the surface expression of PD-L1. The isolated PBMCs were seeded at a density of $1 \times 10^{7}$ cells/well in a six-well plate and incubated with $10 \mu \mathrm{g} / \mathrm{ml}$ phytohemagglutinin (PHA; Roche Diagnostics $\mathrm{GmbH}$, Mannheim, Germany) for $72 \mathrm{~h}$ at $37^{\circ} \mathrm{C}$ to activate T lymphocytes (26). The surviving TNF- $\alpha$ or IFN- $\gamma$ pre-treated, PDLCs were collected and then co-cultured with PHA-activated PBMCs at a ratio of 1:50 for $48 \mathrm{~h}$ for $37^{\circ} \mathrm{C}$; untreated PDLCs were used as a negative control. To inhibit the binding of PD-L1 to PD-1, $10 \mu \mathrm{g} / \mathrm{ml}$ of purified mouse anti-human monoclonal CD274 (PD-L1, B7-H1) antibody (dilution, 1:40; eBioscience, San Diego, CA, USA; cat. no. 14-5983-82) was also added to the TNF- $\alpha$-pre-treated PDLC and PHA-activated PBMC co-culture. Following $18 \mathrm{~h}$ of incubation at $37^{\circ} \mathrm{C}$, the cells were collected and labeled with propidium iodide (PI)/Annexin V-fluorescein isothiocyanate (FITC; Annexin V-FITC Apoptosis Detection kit; Nanjing KeyGen Biotech Co., Ltd., Nanjing, China)/APC-mouse anti-human CD4 monoclonal antibody (dilution, 1:40; BioLegend, Inc.; cat. no. 317416) or PI/annexin V-FITC/APC-mouse anti-human monoclonal CD8 antibody (dilution, 1:40; BioLegend, Inc.; cat. no. 300911) at $4^{\circ} \mathrm{C}$ for $30 \mathrm{~min}$. The labeled cells were subjected to flow cytometric analysis, and the resulting $\mathrm{APC}^{+} / \mathrm{FITC}^{+}$cells were gated to identify apoptotic $\mathrm{T}$ lymphocyte populations.

PDLC survival assay. The PDLCs $\left(1 \times 10^{6}\right)$ pre-treated with $10 \mathrm{ng} / \mathrm{ml}$ TNF- $\alpha$ were stained with carboxyfluorescein diacetate succinimidyl ester (CFSE; Invitrogen; Thermo Fisher Scientific, Inc.) to label the live cells. Briefly, these PDLCs were incubated with CFSE (final concentration $10 \mu \mathrm{M})$ at $37^{\circ} \mathrm{C}$ for $10 \mathrm{~min}$ with gentle agitation. The cells were then washed twice with DMEM supplemented with $10 \%$ FBS and resuspended in DMEM. The CFSE-labeled cells were co-cultured with PHA-treated PBMCs at a ratio of 1:50 for $48 \mathrm{~h}$. PBMCs and PDLCs alone were cultured as CFSE-negative and CFSE-positive controls, respectively. To inhibit the binding of PD-1 to PD-L1, $10 \mu \mathrm{g} / \mathrm{ml}$ of purified anti-human CD274 (PD-L1, B7-H1) antibody was added to the co-culture system at the time of mixing of the two cell types. Following $18 \mathrm{~h}$ of incubation, the cells were collected and stained with PI (final concentration $10 \mu \mathrm{g} / \mathrm{ml}$ ) for $10 \mathrm{~min}$ to label the dead cells. The cells were then subjected to flow cytometric analysis, and signals gated as $\mathrm{CFSE}^{+} / \mathrm{PI}^{-}$were considered live cells.

Establishment of a mouse model of experimental periodontitis, and measurement of the expression levels of PD-LI and PD-1 in the periodontitis tissues. To establish an experimental model or peridontitis, 10 of the mice were randomly selected and were injected with P.g (P.g-injected group); and another 10 mice were injected with PBS as healthy controls (27). All surgery was performed under $200 \mathrm{mg} / \mathrm{kg}$ chloral hydrate [Meryer (Shanghai) Chemical Technology Co., Ltd., Shanghai, China] anesthesia and efforts were made to minimize suffering. For analysis of the outcomes, the severity of periodontitis in the model was categorized into two case types: Case type I was termed mild periodontitis and was defined by the presence of bleeding on probing, furcation cul-de-sac involvement, and facial-lingual tooth movement, without movements in a vertical or mesial direction. Case type II was termed severe periodontitis and was defined by the presence of bleeding on probing, furcation through-and-through involvement, and tooth movement in facial-lingual, vertical and mesial directions.

The mice were sacrificed by cervical dislocation following anesthesia at 10 weeks following the injection, and the inflammatory tissues of the mice were removed. Half of the tissues were embedded in paraffin (Hualin Kangfu, Shanghai, China) for sectioning, followed by immunohistochemical staining of the sections to visualize the expression levels of PD-L1 in the tissues. Single periodontal tissue cells of the inflammatory periodontium were isolated from the other half of the tissues by enzymatic digestion, and the expression levels of PD-L1 and PD-1 on the surface of the cells were analyzed using flow cytometry. Briefly, the tissues were minced with scalpels into sizes $<1 \mathrm{~mm}^{3}$. The minced tissues were then digested with $2 \mathrm{mg} / \mathrm{ml}$ type I collagenase (Sigma-Aldrich) and $100 \mathrm{mg} / \mathrm{ml}$ DNase (Sigma-Aldrich) in PBS at $4^{\circ} \mathrm{C}$ overnight. Finally, the digested tissues were passed through a 200-mesh filter (Yangin Biological, Shanghai, China) to obtain single cells. The spleens of the mice were also removed and passed through the 200-mesh filter to obtain single cells. The cells were stained with rat PE-anti-mouse PD-L1 (3 $\mu$ l per sample; dilution, 1;40; BioLegend, Inc.; cat. no. 124304) or APC-rat anti-mouse PD-1 antibodies (3 $\mu$ l per sample; dilution, 1:40; BioLegend, Inc.; cat. no. 135209), and the expression levels of PD-L1 and PD-1 were determined using flow cytometric analysis.

Immunohistochemistry. Immunohistochemical analyses were performed, as described by Karlsson et al (28). The paraffin-embedded specimens were sliced into 4-5 $\mu \mathrm{m}$-thick sections. The tissue sections were deparaffinized in xylene (Shanghai Macklin Biochemical Co., Ltd., Shanghai, China) for 3-10 min and dehydrated through a series of graded alcohols $(100,100,95$ and $80 \%)$ to displace the water. All sections were treated with $100 \%$ methanol containing $0.3 \%$ $\mathrm{H}_{2} \mathrm{O}_{2}$ for 15 min to block any endogenous peroxidase activity. The tissue sections were immersed in $50 \mathrm{ml} 10 \mathrm{mM}$ citrate buffer ( $\mathrm{pH}$ 6.0) and placed in a microwave oven. Antigens were retrieved by microwaving in citrate buffer $(\mathrm{pH}$ 6.0) for three 6-min cycles. Bovine serum albumin (ZSGB-BIO) was used to block nonspecific IgG bindings. The sections were incubated with rabbit anti-mouse PD-L1 polyclonal antibodies (dilution, 1:200; Abcam, Cambridge, MA, USA; cat. no. ab58810) overnight at $4^{\circ} \mathrm{C}$, followed by incubation with biotinylated goat anti-rabbit secondary antibodies (1:5,000; ZSGB-BIO, Beijing, China) for $30 \mathrm{~min}$ at room temperature, and with streptavidin-peroxidase complex solution for another $30 \mathrm{~min}$ at room temperature. The slides were stained with 3, 3'-diaminobenzidine (Beijing Solarbio Science \& Technology Co., Ltd., Beijing, China). Finally, the sections were counterstained with hematoxylin and observed under an optical microscope (BX51TR; Olympus Corporation, Tokyo, Japan). Image-Pro Plus version 6.0 (Media Cybernetics, Inc., USA) was used for image analysis. 
A
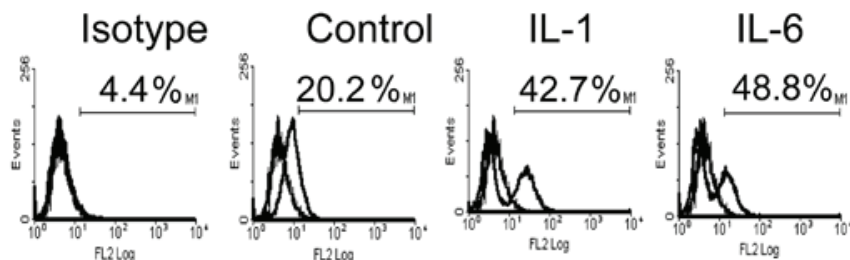

TNF- $\alpha$

IFN-Y

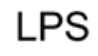

Combination
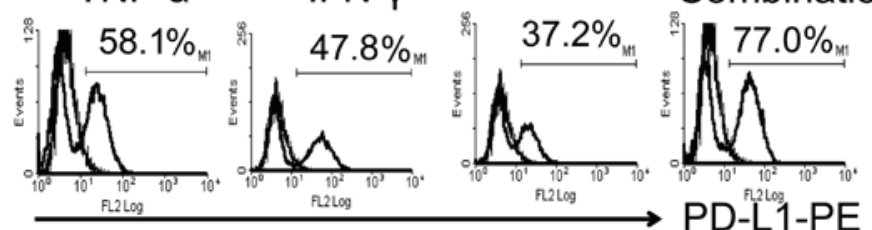

B

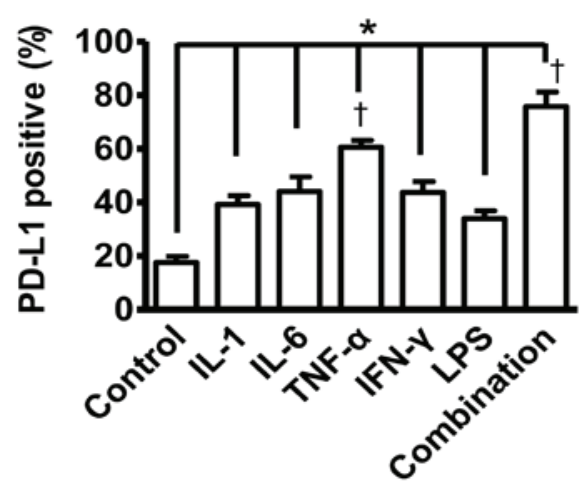

Figure 1. Inducible expression of PD-L1 on PDLCs by inflammatory cytokines. (A) Flow cytometry histogram overlays of PDLCs stimulated with a series of inflammatory cytokines. (B) Comparison of the induced expression of PD-L1. Data is presented as the mean \pm standard error of the mean of three independent experiments. All experiments showed a significant increase in expression levels of PD-L1 ("P<0.05, vs. control). The TNF- $\alpha$ and the combined cytokine groups induced significantly higher expression levels of PD-L1, compared with the other cytokines assessed ( $\mathrm{P}<0.05)$.

Statistical analysis. Statistical analysis was performed using SPSS 17.0 software (SPSS, Inc., Chicago, USA). All values are presented as the mean \pm standard error of the mean. Data were analyzed by one-way analysis of variance, followed by Bonferroni's test. $\mathrm{P}<0.05$ was considered to indicate a statistically significant difference.

\section{Results}

Surface expression of PD-L1 on PDLCs is induced by inflammatory cytokines. In the present study, PDLCs were stimulated with five inflammatory cytokines that are present in the inflammatory microenvironment of the body, including IL-1, IL-6, TNF- $\alpha$, IFN- $\gamma$ and LPS, to examine the inducible expression of PD-L1. As shown in Fig. 1, the expression of PD-L1 was upregulated by IL-1, IL-6, TNF- $\alpha$, IFN- $\gamma$ and LPS, and by the combination of IL-1, IL-6, TNF- $\alpha$ and IFN- $\gamma$, compared with the unstimulated control.

Surface expression of PD-L1 on PDLCs is induced by periodontal pathogens. The inflammatory cytokines secreted by immune and non-immune cells are known to be involved in the destruction of periodontal tissues, and are inducible upon bacterial infection (29). Therefore, the present study examined whether infection with periodontal bacteria induces the expression of PD-L1 on PDLCs. For this assessment, three strains of extensively investigated periodontal pathogens, including P.g, F.n and P.I, were selected for co-culture with the PDLCs for $18 \mathrm{~h}$, followed by flow cytometric analyses of the surface
$\mathbf{A}$
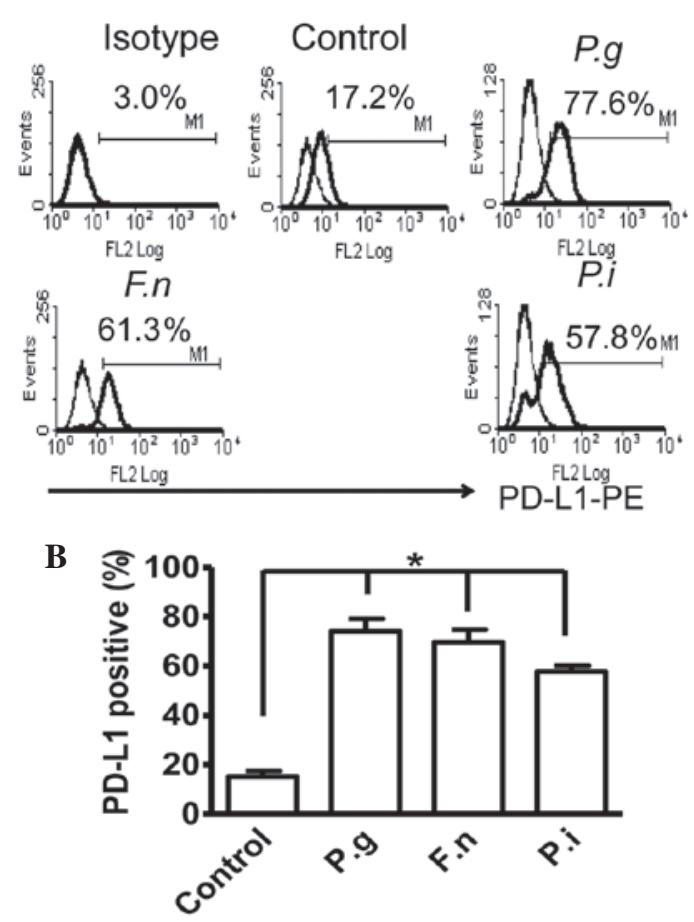

Figure 2. Common periodontal pathogens induce the expression of PD-L1 on PDLCs. (A) Flow cytometry histogram overlays of PDLCs co-cultured with P.g, F.n and P.i. (B) Comparison of expression levels of PD-L1 induced by P.g, F.n and P.i. Data are expressed as the mean \pm standard error of the mean of three independent experiments. All three strains significantly increased the expression of PD-L1 on the PDLCs, ${ }^{*} \mathrm{P}<0.05$. P.g, Porphyromonas gingivalis; F.n, Fusobacterium nucleatum; P.i, Prevotella intermedia; PD-L1, programmed death 1 ligand 1; PDLCs, periodontal ligament cells. 


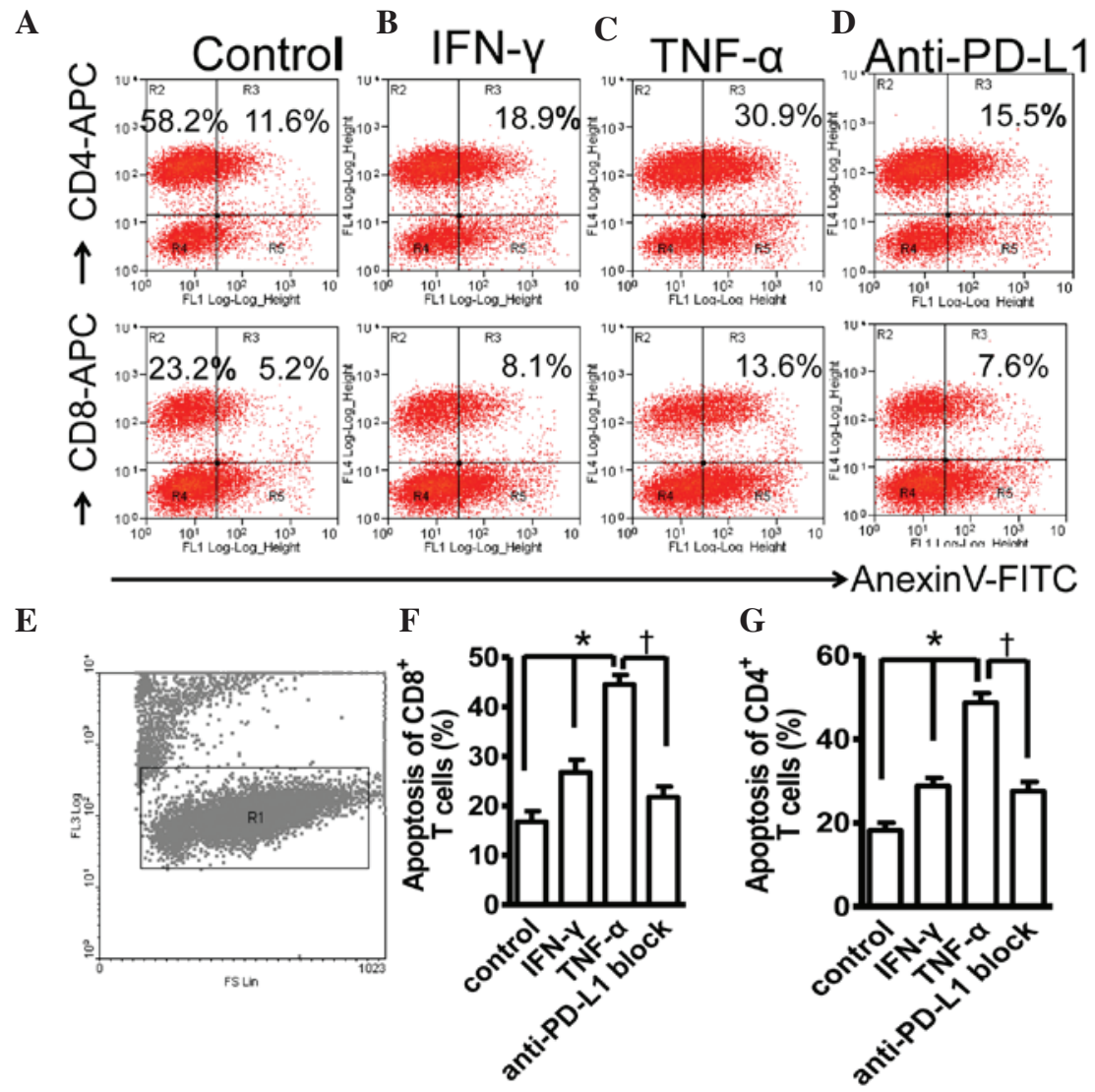

Figure 3. TNF- $\alpha$ - and IFN- $\gamma$-induced expression of PD-L1 on PDLCs caused apoptosis of activated T cells. (A) Two-color flow cytometry histograms of activated PBMCs co-cultured with untreated PDLCs. PDLCs pretreated with (B) IFN- $\gamma$ or (C) TNF- $\alpha$, or incubated with (D) TNF- $\alpha$-pretreated PDLCs and anti-PD-L1 antibody. (E) PI- cells were gated in R1 to exclude necrotic cells. Comparison of (F) CD8 ${ }^{+}$and (G) CD4 ${ }^{+}$T cell apoptosis induced by TNF- $\alpha$ and IFN $-\gamma$, and in the presence of anti-PDL1 antibodies. Data are presented as the mean \pm standard error of the mean of three independent experiments. The increases in T cell apoptosis caused by pretreatment with TNF- $\alpha$ or IFN- $\gamma$ were statistically significant ("P<0.05), and the presence of anti-PD-L1 antibodies caused a considerable reduction in the fraction of apoptotic T cells ( $\mathrm{P}<0.05$ ). PD-L1, programmed death 1 ligand 1; PDLCs, periodontal ligament cells; IFN- $\gamma$, ibterferon- $\gamma$; TNF- $\alpha$, tumor necrosis factor- $\alpha$; APC, antigen-presenting cell; PI, propidium iodide; FITC, fluorescein isothiocyanate.

A B

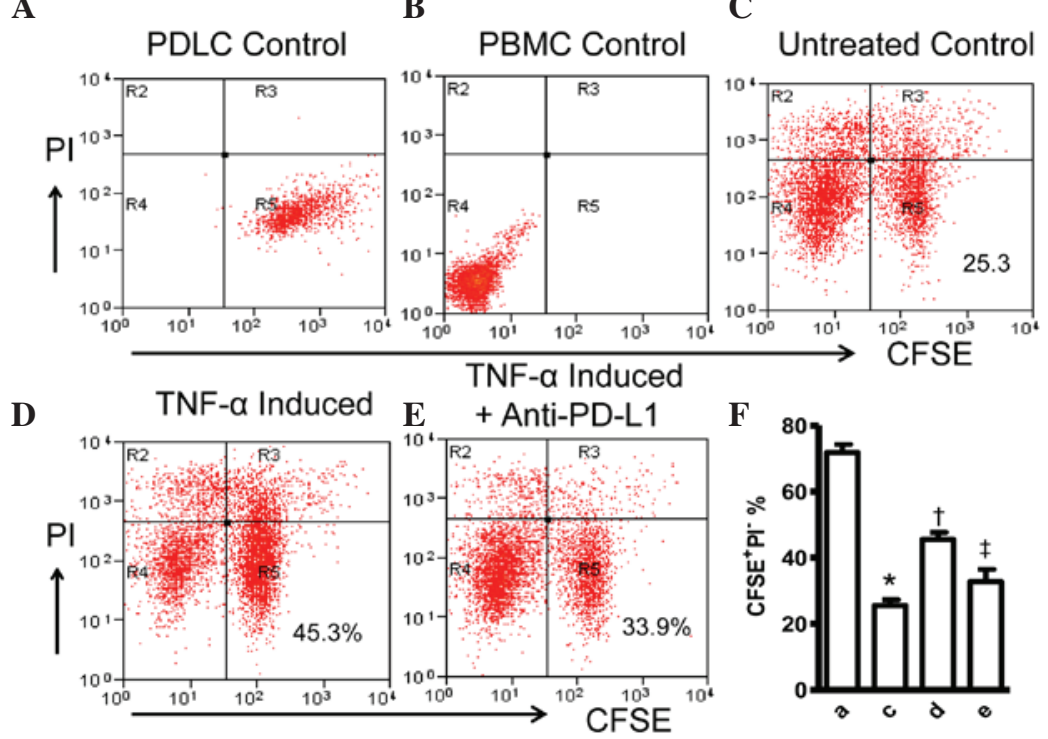

Figure 4. Expression of PD-L1 on PDLCs improves survival of PDLCs. Flow cytometry histrograms of (A) PDLCs, (B) PHA-activated PBMCs, (C) PDLCs co-cultured with activated PBMCs, (D) PDLCs pretreated with TNF- $\alpha$ and co-cultured with activated PBMCs, and (E) PDLCs pretreated with TNF- $\alpha$, and incubated with activated PBMCs and anti-PD-L1 antibodies. (F) Comparison of PDLC survival, according to the percentages of CFSE ${ }^{+} / \mathrm{PI}^{-}$cells. a, $\mathrm{c}, \mathrm{d}$ and $\mathrm{e}$ represent the PDLC control, untreated control, TNF- $\alpha$ induced and TNF- $\alpha$ induced+anti-PD-L1 groups, respectively. Data are expressed as the mean \pm standard error of the mean of three independent experiments. Co-culturing the activated PMBCs with untreated PDLCs resulted in a significant decrease in viable PDLCs $\left({ }^{*} \mathrm{P}<0.05\right) .{ }^{\dagger} \mathrm{P}<0.05$, significant increase in the viability of the PDLCs following pretreatment with TNF- $\alpha$. The addition of anti-PD-L1 antibody caused a significant decrease in the viability of the PDLCs pretreated with TNF- $\alpha\left({ }^{\$} \mathrm{P}<0.05\right)$. PD-L1, programmed death 1 ligand 1 ; PDLCs, periodontal ligament cells; PMBCs, peripheral blood mononuclear cells; PI, propidium iodide; CFSE, carboxyfluorescein diacetate succinimidyl ester; TNF- $\alpha$, tumor necrosis factor- $\alpha$. 
A

Healthy

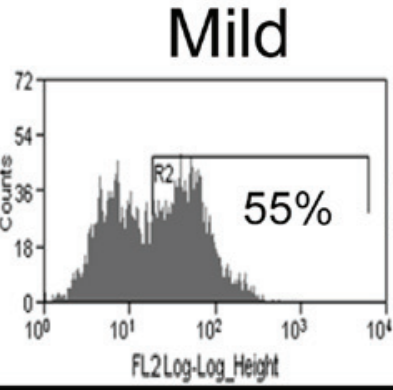

Severe

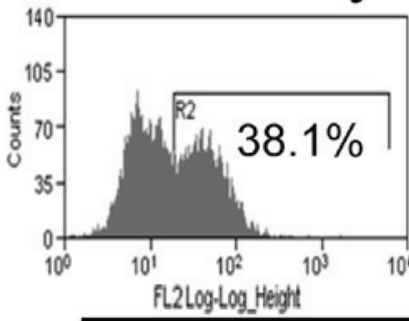

B
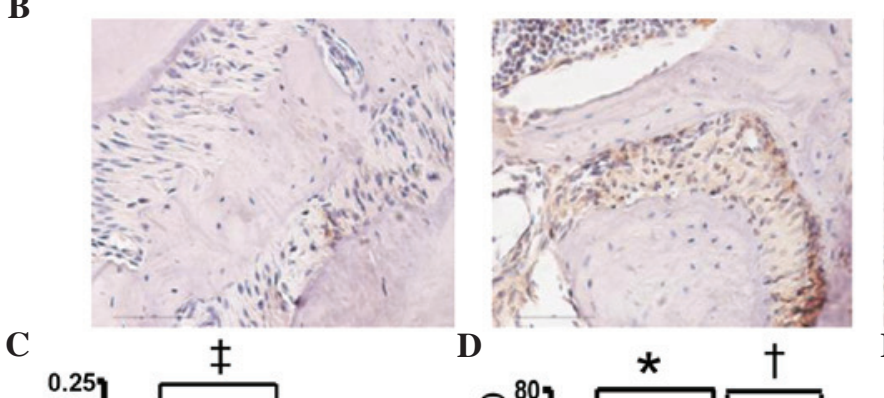

E
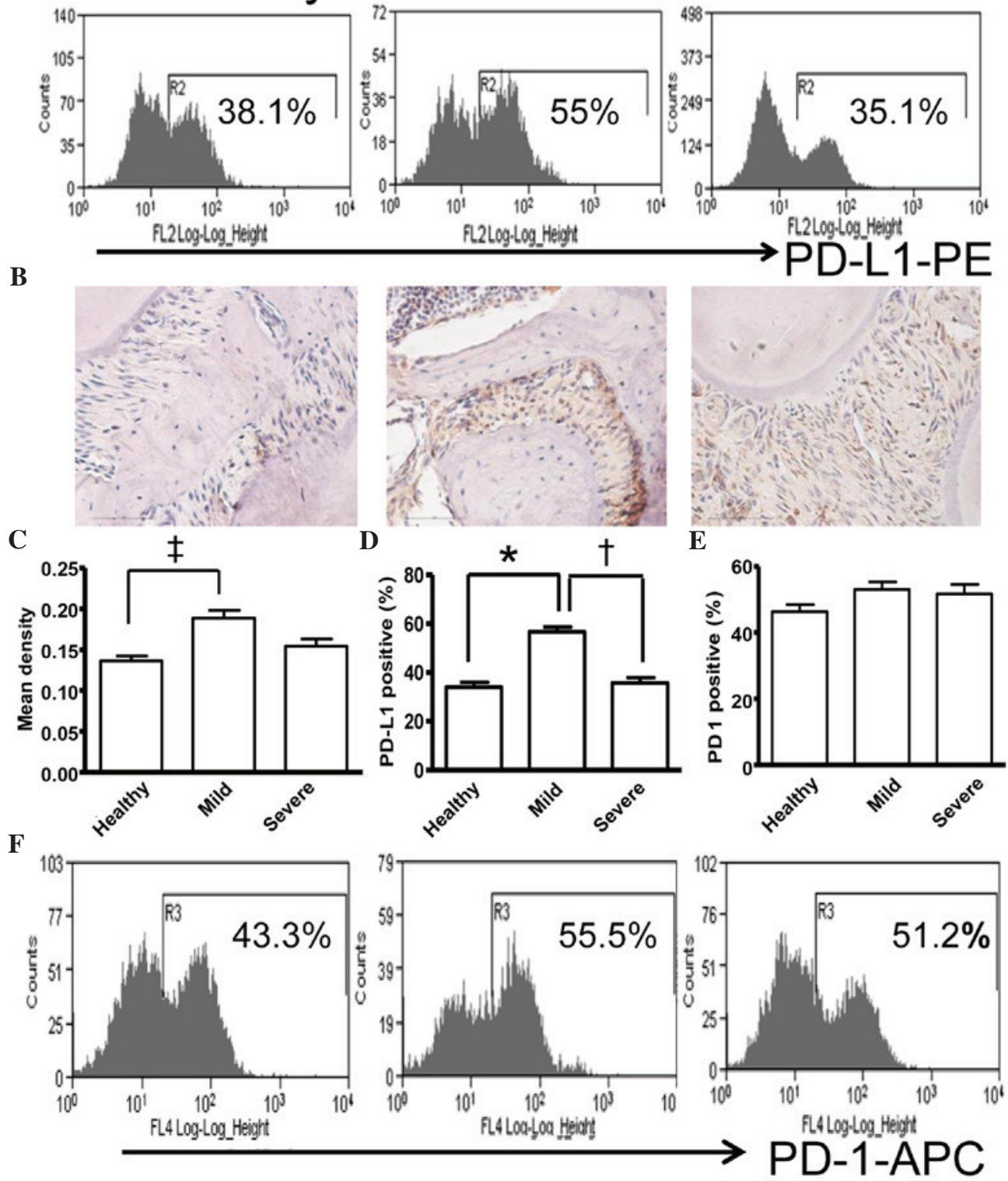

Figure 5. Expression of PD-L1 is correlated with the severity of periodontitis in the experimental periodontitis model. (A) Flow cytometry histograms of the expression of PD-L1 on the surface of periodontal tissue cells from healthy mice, and mice with mild periodontitis and severe periodontitis. (B) Expression of PD-L1 in periodontal tissues from the three groups, detected by immunohistochemical staining (magnification, $\mathrm{x} 400)$. (C) Comparison of the expression of PDL-1 in periodontal tissues between the three groups ( ${ }^{\mathrm{P}}<0.01$, compared with the healthy control). (D) Comparison of the expression of PD-L1 on the surface of the periodontal tissue cells in the three groups $\left({ }^{*} \mathrm{P}<0.05\right.$, compared with the healthy control; ${ }^{\dagger} \mathrm{P}<0.05$, between the mild and severe periodontitis groups). (E) Comparison of the expression of PD-1 on the surface of the periodontal tissue cells between the three groups (F) Flow cytometry histograms of the expression of PD-1 on the surface of periodontal tissue cells from the three groups. No significant differences in the expression of PD-1 were observed between the three groups. Data are expressed as the mean \pm standard error of the mean of three independent experiments. PD-L1, programmed death 1 ligand 1; APC, antigen-presenting cell.

expression of PD-L1. As shown in Fig. 2, the expression of PD-L1 was upregulated by P.g,F.n, and P.i, compared with the unstimulated control.

Expression of PD-L1 on PDLCs causes apoptosis of activated $T$ cells and improves survival of PDLCs. The present study further investigated the molecular function of PD-L1 on PDLCs by co-culturing PHA-activated PBMCs with PDLCs pretreated with TNF- $\alpha$ or IFN- $\gamma$, followed by two-color flow cytometric analysis. The TNF- $\alpha$ - and IFN- $\gamma$-induced expression of PD-L1 were shown to cause significant apoptosis of the activated $\mathrm{CD} 4^{+}$ and $\mathrm{CD}^{+} \mathrm{T}$ lymphocytes. Pretreatment of the PDLCs with
TNF- $\alpha$ led to increases in the percentages of apoptotic CD4 ${ }^{+}$ and apoptotic $\mathrm{CD} 8^{+} \mathrm{T}$ cells, and the addition of IFN- $\gamma$ resulted in increases in the percentages of apoptotic $\mathrm{CD} 4^{+}$and $\mathrm{CD}^{+} \mathrm{T}$ cells (Fig. 3A-D). The PI- cells were gated to exclude necrotic cells (Fig. 3E). In addition, the TNF- $\alpha$-pretreated PDLCs induced higher levels of apoptosis of the $\mathrm{CD} 4^{+}$and $\mathrm{CD} 8^{+} \mathrm{T}$ lymphocytes, compared with the IFN- $\gamma$-pretreated PDLCs. This was consistent with the more marked inducibility of TNF- $\alpha$, compared with IFN- $\gamma$ on the expression of PD-L1, as shown in Fig. 1A. To clarify the cause of the apoptosis, anti-PD-L1 antibodies were added to the cell co-culture at the time of mixing of the PHA-activated PBMCs with TNF- $\alpha$-pretreated PDLCs. 
As shown in Fig. 3C and D, the percentages of apoptosis of the $\mathrm{CD}^{+}$and $\mathrm{CD}^{+} \mathrm{T}$ lymphocytes were reduced significantly, suggesting that the apoptosis of lymphocytes was correlated with the induced expression of PD-L1 on the PDLCs, and that PD-L1 may have negatively regulated the inflammatory responses. The increasing apoptosis of lymphocytes resulting from the upregulation of PD-L1 on the PDLCs inhibited the progression of excessive inflammatory immune responses, and thus reduced the destruction of the PDLCs.

To validate these initial observations of the present study, the cellular effect of the induced expression of PD-L1 on PDLCs was examined by tracking the viability of the PDLCs with CFSE and PI staining. The CFSE-stained PDLCs were co-cultured with PHA-activated PBMCs for $48 \mathrm{~h}$, followed by staining with PI, resulting in a decrease in viable $\left(\mathrm{CFSE}^{+} / \mathrm{PI}^{-}\right)$ PDLCs (Fig. 4A-C). However, the percentage of $\mathrm{CFSE}^{+} \mathrm{PDLCs}$ increased by $20 \%$, compared with the untreated control when the PDLCs were pretreated with TNF- $\alpha$ to induce the expression of PD-L1 prior to co-culturing with the PHA-activated PBMCs (Fig. 4D),. In addition, anti-PD-L1 antibodies were added to the TNF- $\alpha$-pretreated PDLCs and activated PBMCs co-culture at the time of mixing of the two cell cultures, to confirm the cause of cell survival. The percentage of viable PDLCs was reduced to $33.9 \%$, which was not statistically different with that of the untreated control (Figs. 4E and F). These findings further confirmed the protective role of PD-L1 on PDLCs against inflammatory damage.

Expression of PD-L1 is correlated with the severity of periodontitis in the mouse model of experimental periodontitis. A mouse model of experimental periodontitis, exhibiting alveolar bone loss at the maxillary first molar was established in the present study. The expression of PD-L1 in the inflamed periodontium of the mice was analyzed using flow cytometry and immunohistochemistry. As shown in Fig. 5A, the mice with mild periodontitis expressed $\sim 19 \%$ more PD-L1, compared with those with severe periodontitis, indicating that the presence of overexpressed PD-L1 may have ameliorated inflammation in the periodontal tissues, which resulted in periodontitis with less alveolar bone loss. The immunohistochemical staining of the corresponding tissue sections also indicated a considerable increase in the expression of PD-L1 in the periodontal tissues of the mice with mild periodontitis only (Fig. 5B and C). The animal experiments revealed a negative correlation between the expression of PD-L1 and the severity of destruction of the periodontal tissues (Fig. 5D). This is in accordance with the suggested protective role of PD-L1 in immunological damage.

As the PD-L1/PD-1 pathway is known to inhibit $\mathrm{T}$ cell-mediated immune responses (30), the present study also examined the expression levels of PD-1 in the periodontal tissues, peripheral blood and spleen of the periodontitis mouse model. Notably, no significant correlation was found between the expression levels of PD-L1 and PD-1 in the periodontal tissues (Fig. 5E and F) and other tissues (data not shown). This suggested the involvement of an alternative mechanism.

\section{Discussion}

Periodontitis is an inflammatory disease caused by pathogenic oral microbiota. The majority of periodontal microorganisms can destroy tissues either directly through tissue invasion and production of harmful substances, which induce cell death and tissue necrosis, or indirectly through the activation of inflammatory cells, which secrete mediators that act on effectors to destruct periodontal tissues (1,31-34). The infection of periodontal tissues by periodontal pathogens triggers host immune and inflammatory responses to defend the oral tissues against the bacteria $(5,12,31,35)$.

The immune and inflammatory responses initiated by periodontal pathogens can act as a double-edged sword, which may either protect or damage the periodontal tissues. However, in several cases, aberrant host immune responses, rather than pathogen-specific toxins or by-products, are the real pathogenic factors that cause chronic inflammatory diseases or function as risk factors for diseases (18). T cells can modulate bacterium-induced periodontal inflammation and/or alveolar bone destruction (36).

PD-L1 has a crucial immunoregulatory role in the chronicity of inflammatory disorders. The present study demonstrated that PD-L1 was inducibly expressed on PDLCs by inflammatory cytokines and periodontal pathogens. Although Konermann et al (24) reported that the expression of PD-L1 in PDLCs is involved in periodontal immunoinflammatory processes, the function of PD-L1 in periodontitis has received little investigation. Investigating PD-L1 contributes to providing insight into periodontal disease. The dual functions of PD-L1 in regulating $\mathrm{T}$ cell responses have been reported. PD-L1-mediated signals are able to co-stimulate early $\mathrm{T}$ cell priming and differentiation in vivo and in vitro (37), and PD-L1 negatively regulates the function and survival of activated $\mathrm{T}$ cells (38). The results of in vitro experiments were consistent with the latter. The PD-L1/PD-1 pathway are involved in the negative regulation of $\mathrm{T}$ cell responses (39). By contrast, there was no significant difference in the expression of PD-1 between the experimental group and the healthy controls. These data suggested that PD-L1 may inhibit the destruction of periodontal tissues through an alternative way. Further investigations are required to resolve this issue.

The present study analyzed the expression levels of PD-L1 and PD-1 on the surface of cells, which were separated from periodontium. Only the cells of the mild periodontitis group showed increased expression of PD-L1 in the inflamed periodontium. This suggested that the mice assessed had different sensitivities against the same periodontal infection, and expressed different levels of PD-L1. Those with high expression levels of PD-L1 following P.g infection, exhibited a downregulated inflammatory response, avoiding damage to the periodontal tissues, and exhibiting mild periodontitis. By contrast, those expressing lower levels of PD-L1 resulted in severe periodontitis. Although the present study did not identify a direct association between PD-L1 and periodontal tissue destruction, evidence suggests that inflammatory tissue destruction can be inhibited by PD-L1 (40).

In conclusion, the present study provided direct in vitro evidence supporting the role of PDLC-expressed PD-L1 in the inflammatory response against infection with periodontal pathogens. In addition, the results demonstrated a negative correlation between the expression of PD-L1 and the severity of destruction of the periodontal tissues. The results of the present study provides further understanding on the pathogenesis 
of periodontitis and the protective mechanism against bacteria-induced inflammatory damage, and has potential implication on the prevention and treatment of periodontitis.

\section{Acknowledgements}

This study was supported by the National Natural Science Foundation of China (grant no. 81372892).

\section{References}

1. Lindhe J, Ranney R, Lamster I, et al: Consensus report: Chronic periodontitis. Annals of Periodontology 4: 38-38, 1999.

2. Eke PI, Dye BA, Wei L, Thornton-Evans GO and Genco RJ CDC Periodontal Disease Surveillance workgroup: James Beck (University of North Carolina, Chapel Hill,USA), Gordon Douglass (Past President, American Academy of Periodontology), Roy Page (University of Washin): Prevalence of periodontitis in adults in the United States: 2009 and 2010. J Dent Res 91: 914-920, 2012.

3. Bascones-Martínez A, Arias-Herrera S, Criado-Cámara E, Bascones-Ilundáin $\mathrm{J}$ and Bascones-Ilundáin C: Periodontal disease and diabetes. Adv Exp Med Biol 771: 76-87, 2012.

4. Bascones-Martínez A, Muñoz-Corcuera M, Noronha S, Mota P, Bascones-Ilundain C and Campo-Trapero J: Host defence mechanisms against bacterial aggression in periodontal disease: Basic mechanisms. Med Oral Patol Oral Cir Bucal 14: e680-e685, 2009.

5. Genco RJ and Slots J: Host responses in periodontal diseases. J Dent Res 63: 441-451, 1984.

6. de Brito LC, Teles FR, Teles RP, Totola AH, Vieira LQ and Sobrinho AP: T-lymphocyte and cytokine expression in human inflammatory periapical lesions. J Endod 38: 481-485, 2012.

7. Hitzig C and Ciosi P: Periodontitis and the immune system. Actual Odontostomatol (Paris) 33: 573-579, 1979 (In French).

8. Kilian M: Role of the immune system in chronic periodontitis. Tandlaegebladet 83: 123-127, 1979 (In Swedish).

9. Wang LY, Jin Y and Lin XP: The role of adaptive immune response in periodontitis. Zhonghua Kou Qiang Yi Xue Za Zhi 48: 115-118, 2013 (In Chinese).

10. Benakanakere M and Kinane DF: Innate cellular responses to the periodontal biofilm. Front Oral Biol 15: 41-55, 2012.

11. Dixon DR, Bainbridge BW and Darveau RP: Modulation of the innate immune response within the periodontium. Periodontol 2000 35: 53-74, 2004

12. Gemmell E and Seymour GJ: Modulation of immune responses to periodontal bacteria. Curr Opin Periodontol 28-38, 1994.

13. El-Awady AR, Messer RL, Gamal AY, Sharawy MM, Wenger KH and Lapp CA: Periodontal ligament fibroblasts sustain destructive immune modulators of chronic periodontitis. J Periodontol 81: 1324-1335, 2010.

14. Fukushima H, Kajiya H, Takada K, Okamoto F and Okabe K: Expression and role of RANKL in periodontal ligament cells during physiological root-resorption in human deciduous teeth. Eur J Oral Sci 111: 346-352, 2003

15. Choi S, Cho TJ, Kwon SK, Lee G and Cho J: Chondrogenesis of periodontal ligament stem cells by transforming growth factor- $\beta 3$ and bone morphogenetic protein- 6 in a normal healthy impacted third molar. Int J Oral Sci 5: 7-13, 2013.

16. Fujita T, Shiba H, Sakata M, Uchida Y, Nakamura S and Kurihara H: SPARC stimulates the synthesis of OPG/OCIF, MMP-2 and DNA in human periodontal ligament cells. J Oral Pathol Med 31: 345-352, 2002.

17. Freeman GJ, Long AJ, Iwai Y, Borque K, Chernova T, Nishimura H, Fitz LJ, Malenkovich N, Okazaki T, Byrne MC, et al: Engagement of the PD-1 immunoinhibitory receptor by a novel B7 family member leads to negative regulation of lymphocyte activation. J Exp Med 192: 1027-1034, 2000.

18. Dong $\mathrm{H}$ and Chen $\mathrm{X}$ : Immunoregulatory role of $\mathrm{B} 7-\mathrm{H} 1$ in chronicity of inflammatory responses. Cell Mol Immunol 3: 179-187, 2006.

19. Groeger S, Domann E, Gonzales JR, Chakraborty T and Meyle J: B7-H1 and B7-DC receptors of oral squamous carcinoma cells are upregulated by Porphyromonas gingivalis. Immunobiology 216 : $1302-1310,2011$.
20. Zitvogel L and Kroemer G: Targeting PD-1/PD-L1 interactions for cancer immunotherapy. Oncoimmunology 1: 1223-1225, 2012.

21. Chen J, Feng Y, Lu L, Wang H, Dai L, Li Y and Zhang P: Interferon- $\gamma$-induced PD-L1 surface expression on human oral squamous carcinoma via PKD2 signal pathway. Immunobiology 217: 385-393, 2012.

22. Francisco LM, Salinas VH, Brown KE, Vanguri VK, Freeman GJ, Kuchroo VK and Sharpe AH: PD-L1 regulates the development, maintenance, and function of induced regulatory $\mathrm{T}$ cells. J Exp Med 206: 3015-3029, 2009.

23. Lu W, Lu L, Feng Y, Chen J, Li Y, Kong X, Chen S, Li X, Chen $\mathrm{Q}$ and Zhang P: Inflammation promotes oral squamous carcinoma immune evasion via induced programmed death ligand-1 surface expression. Oncol Lett 5: 1519-1526, 2013.

24. Konermann A, Beyer M, Deschner J, Allam JP, Novak N, Winter J, Jepsen S and Jäger A: Human periodontal ligament cells facilitate leukocyte recruitment and are influenced in their immunomodulatory function by Th17 cytokine release. Cell Immunol 272: 137-143, 2012.

25. Arnold LF and Baram P: In vitro culture of periodontal ligament cells. J Dent Res 51: 953-959, 1972.

26. Binns RM, Licence ST, Wooding FB and Duffus WP: Active lymphocyte traffic induced in the periphery by cytokines and phytohemagglutinin: Three different mechanisms? Eur J Immunol 22: 2195-2203, 1992.

27. Polak D, Wilensky A, Shapira L, Halabi A, Goldstein D, Weiss EI and Houri-Haddad Y: Mouse model of experimental periodontitis induced by Porphyromonas gingivalis/Fusobacterium nucleatum infection: Bone loss and host response. J Clin Periodontol 36: 406-410, 2009.

28. Karlsson L, Bondjers C and Betsholtz C: Roles for PDGF-A and sonic hedgehog in development of mesenchymal components of the hair follicle. Development 126: 2611-2621, 1999.

29. Graves DT: The potential role of chemokines and inflammatory cytokines in periodontal disease progression. Clin Infect Dis 28: 482-490, 1999

30. Carter L, Fouser LA, Jussif J, Fitz L, Deng B, Wood CR, Collins M, Honjo T, Freeman GJ and Carreno BM: PD-1: PD-L inhibitory pathway affects both CD4(+) and CD8(+) T cells and is overcome by IL-2. Eur J Immunol 32: 634-643, 2002.

31. Kinane DF and Lappin DF: Immune processes in periodontal disease: A review. Ann Periodontol 7: 62-71, 2002.

32. Alexander MB and Damoulis PD: The role of cytokines in the pathogenesis of periodontal disease. Curr Opin Periodontol 39-53, 1994.

33. Birkedal-Hansen $\mathrm{H}$ : Role of cytokines and inflammatory mediators in tissue destruction. J Periodontal Res 28: 500-510, 1993.

34. Cochran DL: Inflammation and bone loss in periodontal disease. J Periodontol 79 (Suppl 8): S1569-S1576, 2008.

35. Hashim JR, Ruben MP and Kramer GM: Cellular and immune mechanisms in juvenile periodontitis. J West Soc Periodontol Periodontal Abstr 27: 40-47, 1979.

36. Teng YT, Nguyen H, Gao X, Kong YY, Gorczynski RM, Singh B, Ellen RP and Penninger JM: Functional human T-cell immunity and osteoprotegerin ligand control alveolar bone destruction in periodontal infection. J Clin Invest 106: R59-R67, 2000.

37. Subudhi SK, Zhou P, Yerian LM, Chin RK, Lo JC, Anders RA, Sun Y, Chen L, Wang Y, Alegre ML and Fu YX: Local expression of B7-H1 promotes organ-specific autoimmunity and transplant rejection. J Clin Invest 113: 694-700, 2004.

38. Blank C, Kuball J, Voelk1 S, Wiendl H, Becker B, Walter B, Majdic O Gajewski TF, Theobald M, Andreesen R and Mackensen A: Blockade of PD-L1 (B7-H1) augments human tumor-specific T cell responses in vitro. Int J Cancer 119: 317-327, 2006.

39. Liapatas S, Nakou M and Rontogianni D: Inflammatory infiltrate of chronic periradicular lesions: An immunohistochemical study. Int Endod J 36: 464-471, 2003.

40. Scandiuzzi L, Ghosh K, Hofmeyer KA, Abadi YM, Lázár-Molnár E, Lin EY, Liu Q, Jeon H, Almo SC, Chen L, et al: Tissue-expressed B7-H1 critically controls intestinal inflammation. Cell Rep 6: 625-632, 2014. 